\title{
A NOVEL METHOD FOR CALCULATION OF KNEE DEFORMATION ANGLES IN GLINICAL AND SPORT BIOMECHANICS
}

\author{
Ram Haddas \\ Texas Back Institute Research Foundation, Plano, TX, USA \\ Address for correspondence: \\ Dr. Ram Haddas, PhD \\ Texas Back Institute Research Foundation, Plano, TX, USA \\ 6020 W Parker Rd, Suite 200, Plano, TX 75039-8172 \\ E-mail: rhaddas@texasback.com
}

\begin{abstract}
Ahstract. Biomechanical analyses seek to improve understanding of the mechanisms of knee injury and to find ways to reduce knee injury incidence. Many clinical biomechanics researchers use a standard kinematic adopted from Newington Children's Hospital. Biomechanical studies include the knee joint configurations, where joint architecture immutably constrains and guides movement outcomes. Investigators will default to reporting resultant joint deformation without considering the influence of joint architectural configurations on kinematic responses. The purpose of this study was to develop a new joint angular kinematic method that accounts for influence of dynamic joint architectural configuration on deformation values. Twenty subjects performed unloaded dynamic flexion/ extension and $45^{\circ}$ cutting maneuver. The knee deformation angles obtained with the new method proposed were compared with the values that obtained using the standard method. One way repeated measurement ANOVA's was used to compare knee deformation angles values from the standard method that uses a static trial and the new method that uses a dynamic trial. The proposed method distinguishes between dynamic joint architectural configuration and joint deformation. Loaded standard abduction/adduction ( $\beta$ ) and rotation $(\gamma)$ angles were $3.4 \pm 1.8^{\circ}$ and $11.2 \pm 3.8^{\circ}$, respectively. Using the new method, the $\beta$ and $\gamma$ angles decrease to $1.5 \pm 1.4^{\circ}$ $(<0.05)$ and $7.1 \pm 1.8^{\circ}(<0.05)$ during cutting. The new method accounted for dynamic joint architectural configuration produced loaded $\beta$ and $\gamma$ angles that had smaller magnitudes than the standard method, suggesting that previous studies may have overestimated $\beta$ and $\gamma$ angles. Injury management strategies could be influenced by a consideration for dynamic joint architectural configuration. Such a consideration could influence ligament repair strategies. Future studies should account for dynamic configuration when establishing the influence of joint deformation on graft design and appropriate isometry values during reconstruction.
\end{abstract}

Key Worlls: Knee Deformation, Joint Architecture, Knee Injuries, Kinematic, Orthopaedic Surgery, Cutting Maneuver

\section{Introduction}

Knee injuries are common in daily and exercise activities and amount to between $12 \%$ and $19 \%$ of all injuries (Arendt, Dick, 1995; Swenson et al., 2013). Anterior cruciate ligament (ACL) injuries create substantial financial impact with costs related to orthopaedic care and rehabilitation in the United States reaching approximately $\$ 850$ million each year. (Di Stasi, Myer, Hewett, 2013; Griffin, Agel, Albohm, 2000) Additional evidence suggests that when the knee is flexed less than $40^{\circ}$ represents the most unprotected range for ACL tears as consistently 
reported in cadaveric (Kanamori, Woo, Ma, 2000; Markolf, O’Neil, Jackson, 2004), in vivo (Heijne, Fleming, Renstrom, 2004; Pedowitz, O'Connor, Akeson, 2003) and computer simulation (Pandy, Shelburne, 1997; Pflum, Shelburne, \& Torry, 2004) studies of ACL strain or force. Biomechanical analyses seek to improve understanding of the mechanisms of knee injury and to find ways to reduce knee injury incidence (Di Stasi et al., 2013; Haddas, James, Hooper, 2015; Haddas, Yang, Sizer, 2015; Jung et al., 2013; Liu-Barba, Hull, Howell, 2007; Patzkowski et al., 2012). These biomechanical studies usually include analysis of knee joint deformation, where joint architecture immutably constrains and guides movement outcomes (Liu-Barba et al., 2007; Wilson, Feikes, Zavatsky, O'Connor, 2000). Second, they attempt to assess resultant knee joint torques that could produce joint deformation, or changes in the architecture that could change the movement outcomes (Besier, Lloyd, Ackland, Cochrane, 2001; Liu-Barba et al., 2007; Uygur, Richards, Jaric, Freitas, Barlow, 2009).

Deformation defines as segment change in orientation as a result of the application of external stress to it, which cause increase/decrease in angular movement (Schafer, 1987). Unfortunately, investigators will default to reporting resultant joint deformation without considering the influence of joint architectural configurations caused by bone structure, articular cartilage, muscles and ligaments on kinematic responses (Chow, Hemleben, Stokic, 2009; Ferber, Davis, Williams, 2003; MacLean, Davis, Hamill, 2006; Malinzak, Colby, Kirkendall, Yu, Garrett, 2001; Pollard, Davis, Jamill, 2004; Uygur et al., 2009). This shortfall could lead to error in estimating joint kinematic responses that relate to performance and injury.

Cadaveric research supports in-vivo consideration for joint configuration changes during joint kinematic measures (Liu-Barba et al., 2007; Wilson et al., 2000). Several investigators have reported configuration changes in the directions of varus/valgus and internal/external rotation when measuring flexion/extension movements in cadaveric knees (Blankevoort, Huiskes, de Lange, 1988; Liu-Barba et al., 2007; Wilson, Feikes, O'Connor, 1998; Wilson et al., 2000).

Many clinical biomechanics researchers use a standard kinematic method proposed by Davis et al. and Kadaba et al., adopted from Newington Children's and Helen Hayes Hospitals, which serves to define joint kinematic and kinetic responses during human movement (Davis, Ounpuu, Tyburski, Gage, 1991; Kadaba, Ramakrishnan, Wootten, 1990). These investigators adopted a standard method for calculating knee joint angular positions and motions necessary for assessing injury risk. As a preliminary step before collecting any trial in this method, the investigators attempt to collect a standing static trial in order to "zero" the joints angles during subject analyses. This defines the starting place for subsequent movement measurement (Davis et al., 1991; Kadaba et al., 1990).

Using a static trial in order to calculate the resultant joint deformation angle at the knee as determined by the standard method is commonly troubled by an important methodological problem. The standard static trial does not account for lax ligament, cartilage deficiencies, bony abnormalities, and tightness of structures surrounding the joint during dynamic configuration. While traditional method may account for the influence of static joint architectural configuration on deformation angles, they do not account for the influence of dynamic configuration on those same angles. This could lead to error in kinematic calculations involved in basic and science, as well as clinical assessment and management strategy choices in the orthopaedic population.

The knee is a triplanar joint with kinematic coupling between flexion/extension, varus/valgus, and internal/ external rotation (Andriacchi, Koo, Scanlan, 2009). As a consequence of joint architectural configuration, the obliquity of the actual anatomical knee flexion/extension axis relative to the long axes of the thigh and shank produce knee varus/valgus and internal/external rotation angular displacement (Liu-Barba et al., 2007; Wilson et al., 
1998). Additional external loading, which potentially create a torque or stress, during an activity may potentially produce additional varus/valgus and internal/external rotation angular displacement depending on the magnitude of the external loading and the geometry and integrity of the knee joint. In other words, the dynamic knee motion (without any additional external load) may already exhibit varus/valgus and internal/external rotation angles. Thus, any measures of joint architectural configuration and any use of those measures when determining deformation angles must account for the changes in joint axis obliquity through the range of motion that influence a dynamic joint architectural configuration outcome.

Kinematic measurement accuracy is essential when assessing kinematic and kinetic calculations (i.e. joint angular accelerations, joint powers etc.). Small errors in these calculations could lead to larger errors in further calculations germane to biomechanical assessment of the entire lower quarter. Practically, similar errors may change perspectives on ligament graft isometry, design and use of dynamic joint bracing, joint replacement and limb prosthetics (Fitzpatrick, Clary, Laz, Rullkoetter, 2012; Jung et al., 2013; Kent \& Franklyn-Miller, 2011; Patzkowski et al., 2012).

There is a reasonable solution to the problems associated with discounting the influence of dynamic joint architectural configuration on deformation values. Accurate deformation values could be calculated by subtracting the angles of the relatively unloaded dynamic trial from those of the loaded condition. However, the issue is more complex, since the varus/valgus ( $\beta$ Cardan angle) and internal/external rotations ( $\gamma$ Cardan angle) of the knee in the dynamic trial condition should be expected to vary throughout the range of knee flexion/extension, as witnessed in the previously described cadaveric studies (Liu-Barba et al., 2007; Wilson et al., 1998; Wilson et al., 2000). To account for this complexity, a dynamic trial that assesses architectural configuration throughout the entire range of knee motion can be used to establish the dependence of the $\beta$ and $\gamma$ Cardan angles upon the simple knee flexion angle ( $\alpha$ ). Subsequently, these $\beta$ and $\gamma$ angles can be subtracted from those of loaded trials to calculate the $\beta$ and $\gamma$ deformations of the knee that were only due to the external load and not due to the external load and joint architectural configuration.

The purpose of this study was to develop a new joint angular kinematic method that distinguishes between dynamic joint architectural configuration and joint deformation. The proposed method is innovative and unique, in that it is the first to consider the influence of dynamic architectural configuration trial values on calculating knee deformation angles.

\section{Methods}

Twenty subjects (ten male $(1.87 \pm 0.11 \mathrm{~m}, 90 \pm 14 \mathrm{~kg})$ and ten female $(1.67 \pm 0.11 \mathrm{~m}, 69 \pm 19 \mathrm{~kg})$ participated in the investigation. All the subjects were healthy varsity basketball players. All participants read and signed an informed consent form approved by the affiliated university's Institutional Review Board for the protection of human subjects. The subjects performed six slow dynamic trial knee flexion/extension of the right shank on the femur while sitting in open kinetic chain from proximally $90^{\circ}$ to $180^{\circ}$ (full extension). The subjects performed three cutting maneuver which they ran forward 8 meters at a moderate target speed of 5.5-6.5 m/s, stepped on the force platform with the right leg, and executed a $45^{\circ}$ angle cutting maneuver to the left. All trials, the dynamic and the cutting maneuver were averaged. Six Vicon cameras (Oxford Metrics, Ltd., Oxford, United Kingdom) captured the motions of spherical reflective markers attached to the subjects' skin, at a sampling rate of $100 \mathrm{~Hz}$ and calculated 3D coordinates for the markers. The instants of right foot landing and takeoff in the cutting maneuver were determined 
using an AMTI Model OR6-7-1000 force platform embedded in the floor (Advanced Mechanical Technology, Inc., Watertown, MA) synchronized with the Vicon and gathering data at a sampling rate of $1000 \mathrm{~Hz}$. Ten spherical reflective markers were attached to the subjects' skin in the following locations: left and right anterior superior iliac spines (ASIS); left and right posterior superior iliac spines (PSIS); medial and lateral of the femoral epicondyles of the right leg; medial and lateral malleoli of the right leg.

The positions of the ASIS bony landmarks were estimated from the positions of the ASIS marker centers, the distance from each marker center to the corresponding ASIS bony landmark, and a convergence angle in the transverse plane of the pelvis. The distance from the ASIS marker center to the ASIS bony landmark was the sum of the $15.7 \mathrm{~mm}$ distance from the marker center to the skin and $8 \mathrm{~mm}$ of estimated tissue thickness (Bush \& Gutowski, 2003). The right hip joint was estimated to be located at distances of $36 \%, 22 \%$ and $30 \%$ of pelvic width laterally, posteriorly and caudally, relative to the midpoint between the ASIS bony landmarks (Bell, Brand, Pedersen, 1989; Seidel, Marchinda, Dijkers, Soutas-Little, 1995). The right knee was calculated as the midpoint between the medial and lateral knee markers, and the right ankle as the midpoint between the medial and lateral ankle markers. Quintic spline functions were fit to the time-dependent coordinates of each body landmark with a smoothing factor equivalent to a $15 \mathrm{~Hz}$ digital filter (Woltring, 1986).

Two non-inertial right-handed orthogonal reference frames were defined for the thigh $\left(R_{T}\right)$, and shank $\left(R_{S}\right)$ of the right lower extremity. The $3 \mathrm{D}$ orientations of the three axes of $R_{T}$ and $R_{S}$ were calculated for all times of the dynamic trials. The knee configuration was calculated for every normalized time-point of the dynamic trial. This was expressed in terms of the three Cardan angles $\left(\alpha, \beta\right.$ and $\gamma$ ) that would be needed to rotate $R_{S}$ into an orientation identical to that of $\mathrm{R}_{\mathrm{T}}$ in $\alpha-\beta-\gamma$ order of rotations.

The $\alpha, \beta$ and $\gamma$ angles of the shank relative to the thigh were calculated for the loaded cutting conditions. The predicted $\beta$ and $\gamma$ angles of the dynamic trial were then subtracted from the $\beta$ and $\gamma$ angles of the loaded condition to calculate, respectively, the deformations of the knee joint in the directions of varus/valgus $(\beta)$ and internal/external rotation $(\gamma)$ during the loaded trial. The average knee deformation angles between landing to take off obtained with the new kinematic method proposed using a dynamic trial were compared with the average values that would have been obtained using the standard kinematic method with a static trial for each subject. One way repeated measurement ANOVA's were used to compare knee deformation angles values from the standard method that using a static trial and the new method that using a dynamic trial.

\section{Results}

The proposed joint angular kinematic method, using a dynamic trial instead of static trial, distinguishes between dynamic joint architectural configuration and joint deformation. The polynomials of the $\beta$ and $\gamma$ versus simple knee angle (knee flexion) relationships was plotted in the all dynamic trials (Figures 1 and 2). The dynamic trial $\beta$ values showed little intra-subject variability, but $\gamma$ values were more variable as supported by the literature (Table 1) (Blankevoort et al., 1988; Liu-Barba et al., 2007; Wilson et al., 1998; Wilson et al., 2000).

Analysis of the dynamic maneuver with the new kinematic method proposed produces smaller deformation values compared to the standard kinematic method (Table 1, Figure 3). The mean and standard deviation (SD) of static trial abduction/adduction ( $\beta$ ) and internal/external rotation $(\gamma)$ angles were $0.9 \pm 0.7^{\circ}$ and $2.1 \pm 2.0^{\circ}$, respectively. The mean and SD of dynamic trial abduction/adduction $(\beta)$ and internal/external rotation $(\gamma)$ angles were $3.3 \pm 1.9^{\circ}$ and $4.9 \pm 3.2^{\circ}$, respectively. The relationships of $\beta$ and $\gamma$ with simple knee angle (knee flexion) was plotted with all 
Table 1. Descriptive (mean $\pm S D$ ) results for mean knee joint angles using the standard method (using static trial) and the suggested method (using dynamic trial) during a cutting maneuver for all subjects $(n=16)$

\begin{tabular}{|c|c|c|c|c|}
\hline & \multicolumn{2}{|c|}{ Beta } & \multicolumn{2}{|c|}{ Gamma } \\
\hline Static Trial & \multicolumn{2}{|c|}{$0.9 \pm 0.7$} & \multicolumn{2}{|c|}{$2.1 \pm 2.0^{\circ}$} \\
\hline \multirow[t]{2}{*}{ Dynamic Trial } & \multicolumn{2}{|c|}{$3.3 \pm 1.9$} & \multicolumn{2}{|c|}{$4.9 \pm 3.2^{\circ}$} \\
\hline & Beta-Static & Beta-Dynamic & Gamma-Static & Gamma-Dynamic \\
\hline Cutting Maneuver & $3.4 \pm 1.8^{\circ}$ & $1.5 \pm 1.4^{\circ *}$ & $11.2 \pm 3.8^{\circ}$ & $7.1 \pm 1.8^{\circ *}$ \\
\hline
\end{tabular}

${ }^{*} \mathrm{P} \leq 0.05$, significant difference between methods.

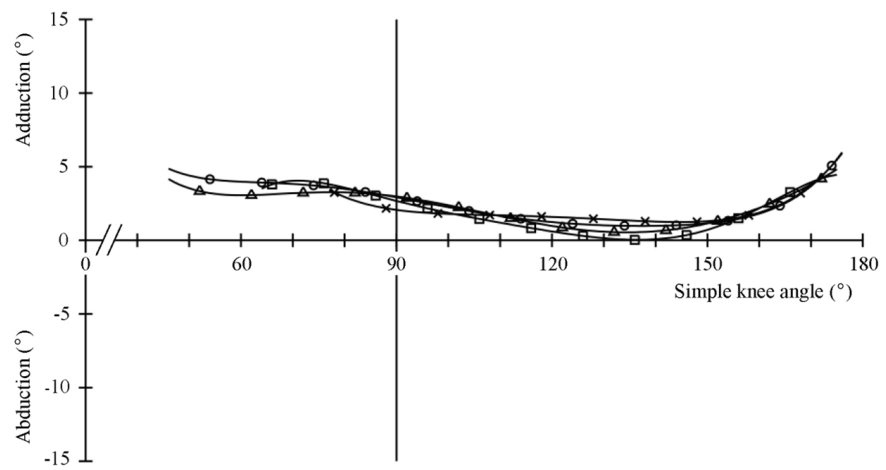

Figure 1. Polynomials of the $\beta$ versus simple knee angle (180 degrees represent full extension) in the relatively unload dynamic trials of a representative subject

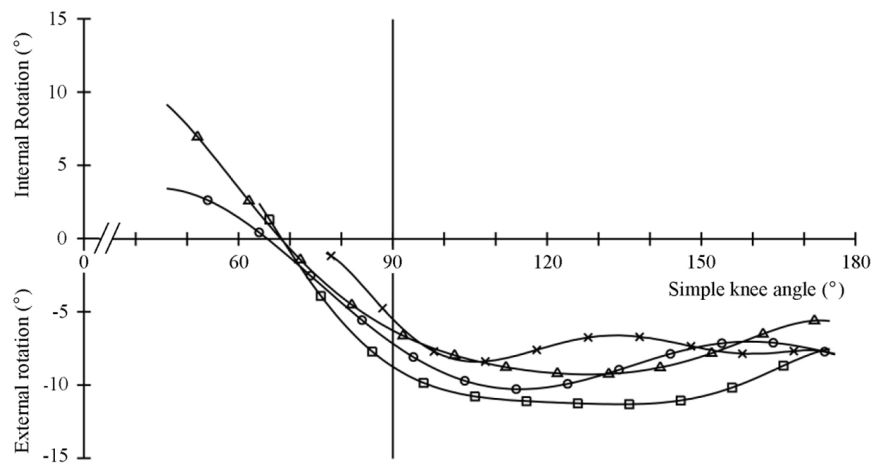

Figure 2. Polynomials of the $\gamma$ versus simple knee angle (180 degrees represent full extension) in the relatively unload dynamic trials of a representative subject 


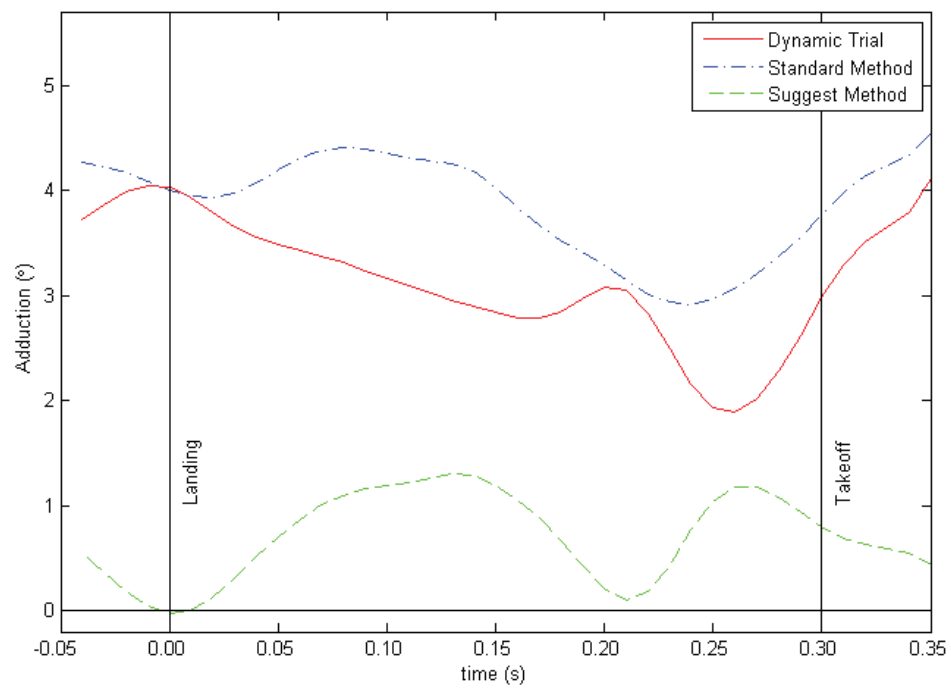

Figulle 3. Knee architecture and knee deformation ( $\beta$ ) using the standard method versus the suggested method during cutting maneuver

available dynamic trials, and the 6 th degree polynomials fitted to the $\beta$ and $\gamma$ angles. Using the standard kinematic method when taking to account the static trial only, loaded standard $\beta$ and $\gamma$ angles were $3.4 \pm 1.8^{\circ}$ and $11.2 \pm 3.8^{\circ}$. Using the new method when taking to account the dynamic trial, the $\beta$ and $\gamma$ angles were $1.5 \pm 1.4^{\circ}(P<0.05)$ and $7.1 \pm 1.8^{\circ}(P<0.05)$ during cutting (Table 1).

\section{Discussion}

The purpose of this study was to develop a new joint angular kinematic method that accounts for the influence of dynamic joint architectural configuration on deformation values using a dynamic trial instead of dynamic one. The new method accounted for dynamic joint architectural configuration produced loaded $\beta$ and $\gamma$ angles that had smaller magnitudes than the standard method. The results of the current analyses suggest that previous studies overestimate $\beta$ and $\gamma$ angles by using the standard kinematic method based on static trials (Chow et al., 2009; Davis et al., 1991; Ferber et al., 2003; Kadaba et al., 1990; MacLean et al., 2006; Pollard et al., 2004; Uygur et al., 2009). By using the standard kinematic method, results reported by previous studies may have failed to exclude dynamic joint architectural configuration when reporting joint deformation values, thus distorting their outcomes.

Our findings support previous cadaveric studies that exhibited a distinction between knee joint architecture and knee deformation angles and reflected structural differences between different knee specimens (Blankevoort et al., 1988; Liu-Barba et al., 2007; Wilson et al., 1998; Wilson et al., 2000). The results of the current study support that dynamic joint architectural configuration in the knee already create varus/valgus and internal/external rotation structural angles (Figures 1 and 2). The $\beta$ and $\gamma$ values, when using a static trial, represent the knee architectural configuration and joint deformation as knee deformation, whereas using a dynamic trial for dynamic joint architectural configuration reduces knee deformation angle outcomes (Figure 3, Table 1). The $\beta$ and $\gamma$ angles 
of the dynamic trial motions demonstrated a moderate tendency to follow patterns similar to those of the loaded activities. Therefore, the $\beta$ and $\gamma$ angles using the new proposed method generally reached less extreme values versus using the standard method.

While considerable attention has been given to the influence of joint deformation on human performance, (Besier etal., 2001; Ferber etal., 2003; MacLean et al., 2006; Malinzak etal., 2001; Pollard et al., 2004) a consideration for dynamic joint architectural configuration on that performance is wanting. The influence of such configurations on joint kinematics and kinetics during high performance and duress must be examined. Any conclusions could fine tune training strategies based on dynamics changes in loading throughout the joint range.

Along to our findings, the new method has several limitations: (1) different dynamic trial position should be considered; (2) we acknowledge limitations associated using the marker set including skin movement, anthropometric model, system tracking error and data smoothing procedure error; and (3) this method should be examined on people with knee pathology.

\section{Conclusion}

The findings from this new kinematic method could potentially improve the understanding of the mechanisms that produce injury. Any overestimation when reporting joint deformation may result in overlooking other underlying factors that influence injury.

Injury management strategies could be influenced by a consideration for dynamic joint architectural configuration. Such a consideration could influence ligament repair strategies. Future studies should account for dynamic configuration when establishing the influence of joint deformation on graft design and appropriate isometry values during reconstruction. To date, investigators have not accounted for dynamic joint architectural configuration in the design and use of dynamic joint bracing and limb prosthetics. Such a consideration could influence the dynamics of the brace or prosthetic articular mechanism, thus influencing design and production. Finally, dynamic joint configuration should be considered when addressing the challenges joint prosthetic design, production and implantation using the proposed new kinematic method for calculating dynamic knee configuration angles.

\section{Acknowledgements}

This is part of Dr. Ram Haddas thesis work at Indiana University, IN, USA.

\section{References}

Andriacchi, T.P., Koo, S., Scanlan, S.F. (2009). Gait mechanics influence healthy cartilage morphology and osteoarthritis of the knee. J Bone Joint Surg Am, 91 Suppl 1, 95-101. DOI: 10.2106/JBJS.H.01408.

Arendt, E., Dick, R. (1995). Knee injury patterns among men and women in collegiate basketball and soccer. Am Orthop Soc for Sports Med, 23 (6), 694-701.

Bell, A.L., Brand, R.A., Pedersen, D.R. (1989). Prediction of hip joint center location from external landmarks. Human Movement Science, 8, 3-16.

Besier, T.F., Lloyd, D.G., Ackland, T.R., Cochrane, J.L. (2001). Anticipatory effects on knee joints loading during running cutting maneuvers. Medicine \& Science in Sports \& Exercise, 33 (7), 1176-1181.

Blankevoort, L., Huiskes, R., de Lange, A. (1988). The envelope of passive knee joint motion. Journal of Biomechanics, 21 (9), 705-720.

Bush, T.R., Gutowski, P.E. (2003). An approach for hip joint center calculation for use in seated posture. Journal of Biomechanics, 36 , 1739-1743. 
Chow, J.W., Hemleben, M.E., Stokic, D.S. (2009). Effect of centerline-guided walking on gait characteristics in healthy subjects. Journal of Biomechanics, 43, 1134-1137.

Davis, R.B., Ounpuu, S., Tyburski, D., Gage, J.R. (1991). A gait analysis data collection and reduction technique. Human Movement Science, 10, 575-587.

Di Stasi, S., Myer, G.D., Hewett, T.E. (2013). Neuromuscular training to target deficits associated with second anterior cruciate ligament injury. J Orthop Sports Phys Ther, 43 (11), 777-792, A771-711. DOI: 10.2519/jospt.2013.4693.

Ferber, R., Davis, I.M., Williams, D.S. (2003). Gender differences in lower extremity mechanics during running. Clinical Biomechanics, 18, 350-357.

Fitzpatrick, C.K., Clary, C.W., Laz, P.J., Rullkoetter, P.J. (2012). Relative contributions of design, alignment, and loading variability in knee replacement mechanics. J Orthop Res, 30 (12), 2015-2024. DOI: 10.1002/jor.22169.

Griffin, L., Agel, J., Albohm, M. (2000). Noncontact anterior cruciate ligament injuries: Risk factors and prevention strategies. J Am Acad Orthop Surg, 8, 141-150.

Haddas, R., James, C.R., \& Hooper, T.L. (2015). Lower extremity fatigue, sex, and landing performance in a population with recurrent low back pain. J Athl Train, 50 (4), 378-384. DOI: 10.4085/1062-6050-49.3.61.

Haddas, R., Yang, J., Sizer, P. (2015). Effects of Gender and Recurrent Low Back Pain on Lifting Style. Central European Journal of Sport Sciences and Medicine, 11 (3): 15-28. DOI:10.18276/cej.2015.3-02.

Heijne, A., Fleming, B., Renstrom, P. (2004). Strain on the anterior cruciate ligament during closed kinetic chain exercises. Med Sci Sports Exerc, 36, 935-941.

Jung, H.J., Kim, J.H., Lee, H.J., Koo, S., Chang, S.H., Jung, Y.B., Lee, S.H. (2013). The isometry of two different paths for remnantpreserving posterior cruciate ligament reconstruction. Knee Surg Sports Traumatol Arthrosc, 21 (5), 1029-1035. DOI: 10.1007/ s00167-012-2111-6.

Kadaba, M.P., Ramakrishnan, H.K., Wootten, M.E. (1990). Measurement of lower extremity kinematics during level walking. Journal of Orthopaedic Research, 8, 383-392.

Kanamori, A., Woo, S., Ma, B. (2000). The forces in the anterior cruciate ligament and knee kinematics during a simulated pivot shift test: A human cadaveric study using robotic technology. Arthroscopy, 16, 633-639.

Kent, J., Franklyn-Miller, A. (2011). Biomechanical models in the study of lower limb amputee kinematics: a review. Prosthet Orthot Int, 35 (2), 124-139. DOI: 10.1177/0309364611407677.

Liu-Barba, D., Hull, M.L., Howell, S.M. (2007). Coupled motions under compressive load in intact and ACL-deficient knees: a cadaveric study. Journal of Biomechanical Engineering, 129 (6), 818-824.

MacLean, C., Davis, I.M., Hamill, J. (2006). Influence of a custom foot orthotic intervention on lower extremity dynamics in healthy runners. Clin Biomech (Bristol, Avon), 21 (6), 623-630. DOI: 10.1016/j.clinbiomech.2006.01.005.

Malinzak, R.A., Colby, S.M., Kirkendall, D.T., Yu, B., Garrett, W.E. (2001). A comparison of knee joint motion patterns between men and women in selected athletic tasks. Clinical Biomechanics, 16, 438-445.

Markolf, K., O'Neil, G., Jackson, S. (2004). Effects of applied quadriceps and hamstrings muscle loads on forces in the anterior and posterior cruciate ligaments. The Am J Sport med, 32, 1144-1149.

Pandy, M., Shelburne, K. (1997). Dependence of cruciateligament loading on muscle forces and external load. J Biomech, 30, 1015-1024.

Patzkowski, J.C., Blanck, R.V., Owens, J.G., Wilken, J.M., Kirk, K.L., Wenke, J.C., Hsu J.R. Skeletal Trauma Research. Comparative effect of orthosis design on functional performance. J Bone Joint Surg Am, 94 (6), 507-515. DOI: 10.2106/JBJS.K.00254.

Pedowitz, R., O'Connor, J., Akeson, W. (2003). Daniel's Knee Injuries: Ligament and Cartilage Structure, Function, Injury and Repair (2nd ed.). Philadelphia, PA: Lippincott, Williams \& Wilkins.

Pflum, M., Shelburne, K., Torry, M. (2004). Model prediction of anterior cruciate ligament force during drop-landings. Med Sci Sports Exerc, 36, 1949-1958.

Pollard, C.D., Davis, I.M., Jamill, J. (2004). Influence of gender on hip and knee mechanics during a randomly cued cutting maneuver. Clinical Biomechanics, 19, 1022-1031.

Schafer, R.C. (1987). Clinical Biomechanics: Musculoskeletal Actions and Reactions: Williams \& Wilkins.

Seidel, G.K., Marchinda, D.M., Dijkers, M., Soutas-Little, R.W. (1995). Hip joint center location from palpable bony landmarks a cadaver study. Journal of Biomechanics, 28, 995-998. 
Swenson, D.M., Collins, C.L., Best, T.M., Flanigan, D.C., Fields, S.K., Comstock, R.D. (2013). Epidemiology of knee injuries among us high school athletes, 2005/06-2010/11. Med Sci Sports Exerc, 45 (3), 462-469.

Uygur, M., Richards, J.G., Jaric, S., Freitas, P.B., Barlow, D.A. (2009). Kinematics and kinetics of unanticipated misstep condition: Femoral fracture implication in the elderly. Journal of Biomechanics, 42, 1241-1245.

Wilson, D.R., Feikes, J.D., O'Connor, J.J. (1998). Ligaments and articular contact guide passive knee flexion. Journal of Biomechanics, 31 (12), 1127-1136.

Wilson, D.R., Feikes, J.D., Zavatsky, A.B., O'Connor, J.J. (2000). The components of passive knee movement are coupled to flexion angle. Journal of Biomechanics, 33 (4), 465-473.

Woltring, H.J. (1986). A fortran package for generalized, cross-validation spline smoothing and differentiation. Advanced Engineering Software, 8, 104-113.

Cite this anticle as: Haddas, R. (2016). A Novel Method for Calculation of Knee Deformation Angles in Clinical and Sport Biomechanics. Central European Journal of Sport Sciences and Medicine, 13 (1), 5-13. DOI: 10.18276/cej.2016.1-01. 
